\title{
Non Resistive Analysis of Rotational Instabilities in FRC and the Unicamp TC-1 $\mathrm{m}=4$ Results
}

\author{
M. A. M. Santiago \\ Instituto de Física, Universidade Federal Fluminense \\ 24210-340, Niterói, Rio de Janeiro, Brazil \\ A. S. Assis \\ Instituto de Matematica, Universidade Federal Fluminense \\ 24020-100, Niteroi, Rio de Janeiro, Brazil \\ C. A. de Azevedo \\ Instituto de Física, Universidade do Estado do Rio de Janeiro \\ 20550-013, Rio de Janeiro, RJ, Brazil \\ P. H. Sakanaka, M. Machida, E. Aramaki, L. A. Berni, \\ D. O. Campos and R. T. Farias \\ Instituto de Física 'Gleb Wataghin' \\ Universidade Estadual de Campinas, UNICAMP \\ 13083-970, Campinas, São Paulo, Brazil
}

Received September 30, 1997

\begin{abstract}
Ideal one dimensional MHD (non-resistive) equations are used to study the rotational instability in field reversed configuration plasmas. Instead of using resistive boundary layer analysis, the eigenmode non hermitian equations are solved on the complex $\omega$-plane using a numerical code constructed using "Mathematica". We take into account the plasma compressibility and compare our results with the Compact Torus (TC-1) experiment of the Universidade Estadual de Campinas (UNICAMP), which is presented here. The $m=4$ rotational mode observed in TC-1 is used to verify the consistency of our model.
\end{abstract}

\section{Introduction}

The prospect of field reversed configuration (FRC) devices to be competitive with other fusion devices relies on the understanding of the stability properties[1][3]. Frieman and Rotenberg [4] developed the original perturbation theory for a flowing equilibrium including the plasma compressibility. We use their basic operator equation to study the rotational mode in field reversed configuration. Other previous work are also concentrated on rotational modes [5]-[8]. According to boundary layer analysis [9],[10], the plasma is governed by ideal MHD with real frequency $\omega$ on both sides of the singular layer $r_{0}$ where the equilibrium magnetic field vanishes. To overcome the accumulation point of the frequency spectrum of the eigenmode, resistivity was introduced in the boundary layer which leads to a growth rate strictly related to resistivity. But previous works [5],[6], indicated that resistivity is not relevant to analyse rotational modes.

Here we use the non resistive model in cylindrical geometry with realistic equilibrium profiles for the density, pressure and magnetic field configuration. We solve the eigenmode equation developed by Frieman [4], using a numerical code constructed with the software "Mathematica" and compare our results with the Compact Torus (TC-1) experiment of UNICAMP, where a $\mathrm{m}=4$ rotational mode is observed. 


\section{Equilibrium and perturbed equations}

We consider a reversed field $\theta$-pinch configuration with axial magnetic field and azimuthal rotation $\Omega$. All variables depend on $r$ only. The equilibrium profiles are given by (see Figures (1) - (3))

$$
\vec{B}=B_{z}(r) \hat{z},
$$

where $B_{z}$ is the magnetic field and is modeled, to be realistic with the TC-1 machine, as

$$
B_{z}(r)=\alpha+(1-\alpha) \tanh \left[(0.55 r)^{4}+(0.55 r)^{2}\right],
$$

and

$\alpha=-\tanh \left[(0.55)^{4}+(0.55)^{2}\right] /\left(1-\tanh \left[(0.55)^{4}+(0.55)^{2}\right]\right)$,

$$
\rho(r)=\left\{\exp \left[-(r / 2)^{8}\right]+0.05\right\} / 1.05
$$

where $\rho$ is the mass density,

$$
\vec{v}=v(r) \hat{\theta}=r \Omega \hat{\theta},
$$

$\vec{v}$ is the velocity field, and the rotational frequency $\Omega$ is assumed to be constant.

We use the non resistive MHD and Maxwell equations, which are given by

$$
\begin{aligned}
\rho \frac{d \vec{v}}{d t} & =-\nabla p+\vec{J} \times \vec{B}, \\
\frac{\partial \rho}{\partial t} & =-\nabla \cdot(\rho \vec{v}),
\end{aligned}
$$

and

$$
\frac{\partial \vec{B}}{\partial t}=\nabla \times(\vec{v} \times \vec{B})
$$

with $\nabla \times \vec{B}=\vec{J}$, where $\vec{J}$ is the current density, $p / \rho^{\gamma}=$ const, $\gamma$ is the ratio of specific heats, and $\nabla \cdot \vec{B}=0$.

In equilibrium $\left(\frac{\partial}{\partial t} \sim 0\right)$, we obtain

$$
\frac{d}{d r}\left(p+B_{z}^{2} / 2\right)=\rho \Omega^{2} r .
$$

We use the Lagrangian representation, linearize the equations and introduce the displacement vector $\vec{\xi}$, which is considered a small quantity given by

$$
\vec{r}=\vec{r}^{0}+\vec{\xi}\left(\vec{r}^{0}, t\right)
$$

where $\vec{r}^{0}$ describes the equilibrium trajectory and $\vec{\xi}(\vec{r}, t)$ describes the displacement from equilibrium, and consider the equilibrium quantities as time independent.

Following the linearization of the basic equations described in [4] we obtain the non hermitian linearized equation of motion in the form

$$
\rho\left(\frac{\partial^{2} \vec{\xi}}{\partial t^{2}}\right)+2 \rho \vec{v} \cdot \nabla\left(\frac{\partial \vec{\xi}}{\partial t}\right)=\vec{F}(\vec{\xi})
$$

where

$$
\begin{aligned}
& \vec{F}(\vec{\xi})=\nabla(\gamma p \nabla \cdot \vec{\xi}+\vec{\xi} \cdot \nabla p-\vec{B} \cdot \vec{Q}) \\
& +\quad \vec{B} \cdot \nabla \vec{Q}+\vec{Q} \cdot \nabla \vec{B}+\nabla \cdot(\rho \vec{\xi} \vec{v} \cdot \nabla \vec{v}-\rho \vec{v} \vec{v} \cdot \nabla \vec{\xi})(10) \\
& \text { and } \quad \vec{Q}=\nabla \times(\vec{\xi} \times \vec{B}) .
\end{aligned}
$$

Assuming normal mode solutions of the form

$$
\vec{\xi}\left(\vec{r}^{0}, t\right)=\vec{\xi}\left(\vec{r}^{0}\right) \exp (i \omega t),
$$

the equation of motion becomes

$$
-\omega^{2} \rho \vec{\xi}+2 i \omega \rho \vec{v} \cdot \nabla \vec{\xi}=\vec{F}(\vec{\xi})
$$

This non Hermitian eigenvalue equation will be solved with appropriate boundary conditions, $\vec{\xi}(r)=0$, for $r=0$ and $r=a$ through a numerical code developed using the software "Mathematica". The pressure $p$ is obtained solving equation (7) numerically.

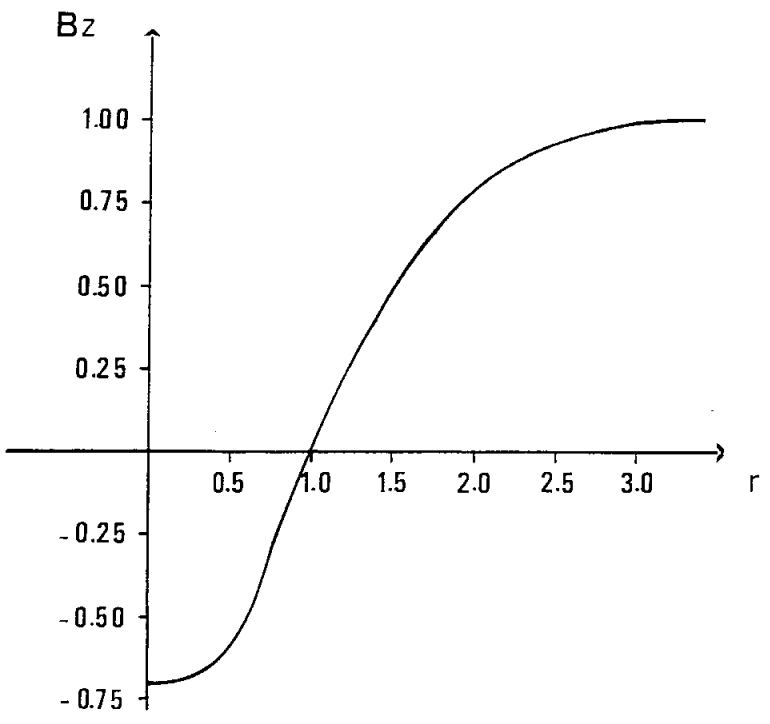

Figure 1. Equilibrium magnetic field profile Bz(r). 


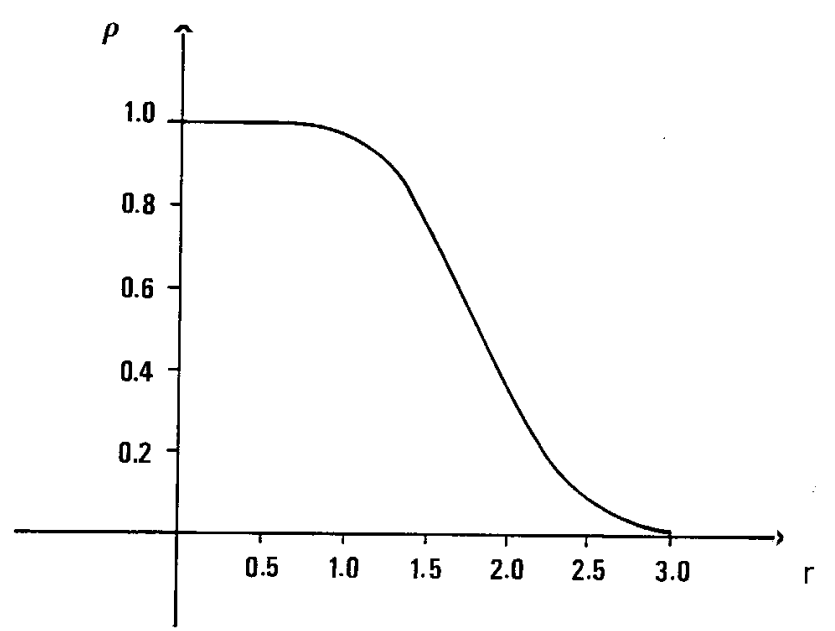

Figure 2. Equilibrium mass density profile $\rho(\mathrm{r})$.

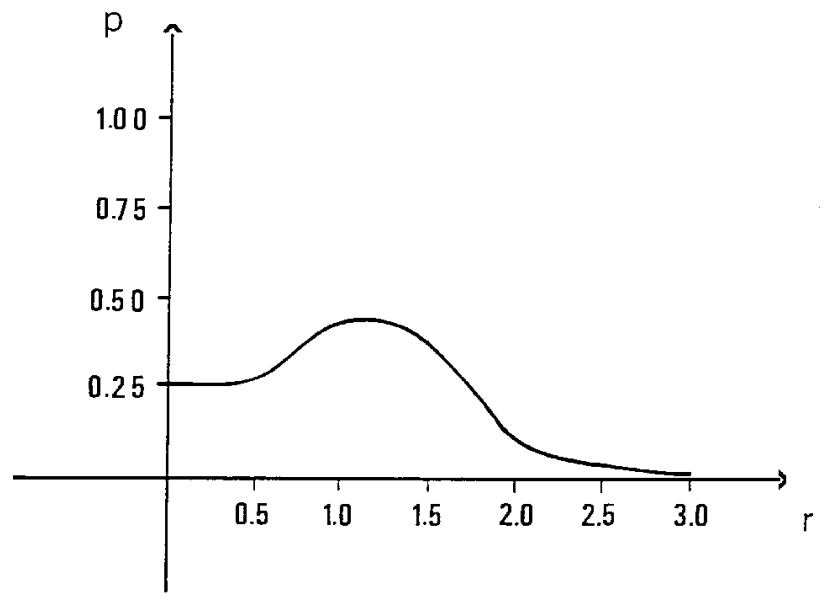

Figure 3. Equilibrium pressure profile $\mathrm{p}(\mathrm{r})$.

\section{The UNICAMP TC-1 results}

The Compact Torus (TC-1) machine at UNICAMP, [11], is a field reversed $\theta$-pinch designed to study FRC formation and stability. The main solenoid is $45 \mathrm{~cm}$ long and $16 \mathrm{~cm}$ in diameter, and the two mirror field solenoids are $10 \mathrm{~cm}$ long and $15 \mathrm{~cm}$ in diameter. The base pressure of $1.0 \times 10^{-6}$ Torr is filled with Hydrogen at $(1$ to 10$) \times 10^{-3}$ Torr. The $7 \mathrm{kV}, 10.8 \mathrm{~kJ}$ polarization capacitor bank produces a reverse bias of $1.0 \mathrm{kG}$. The $25 \mathrm{kV}, 0.5 \mathrm{~kJ}$ preionization capacitor bank with crowbar ionizes partially the working gas and is interrupted after few oscillations, when the $22 \mathrm{kV}, 8.8 \mathrm{~kJ}$ main capacitor bank, with rise time of $5 \mu \mathrm{s}$, is switched on. The main field decays after the main crowbar is about $30 \mu \mathrm{s}$, and the peak magnetic field is $3.6 \mathrm{kG}$.
The typical plasma parameters of the TC- 1 are the following: separatrix radius of $3.5 \mathrm{~cm}$, measured by excluded-flux loops, ion temperature of $180 \mathrm{eV}$, measured by $C I V$ and SiIV impurity lines, electron density of $1.0 \times 10^{15} \mathrm{~cm}^{-3}$ measured by $\mathrm{CO}_{2}$ laser interferometry and electron temperature of $100 \mathrm{eV}$ estimated by pressure balance. The TC-1 machine and its diagnostic set up are shown in Fig. 4.

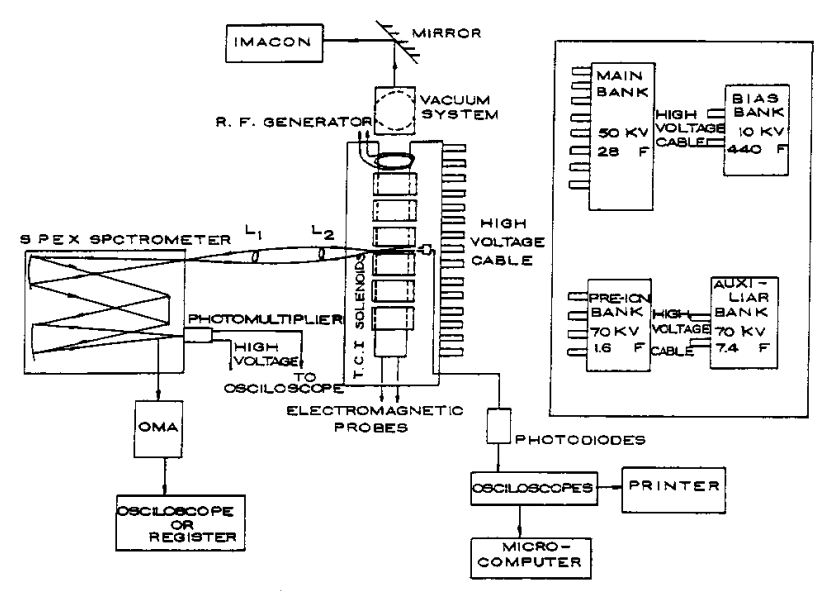

Figure 4. Experimental arrangement and diagnostics of the TC-1 machine in the Plasma Laboratory of UNICAMP.

One feature of the TC-1 machine is the capability of crowbar on preionization bank, which controls the ionization state before the main phase starts, and avoids the normal oscillations from preionization bank to be present after the start of the main discharge bank.

In Fig. 5, we present end-on framing pictures of implosion and equilibrium phases for filling pressure of $3.3 \times 10^{-3}$ Torr and preionization time of $8 \mu \mathrm{s}$ (one period), where usual $m=0$ mode plasma is obtained.

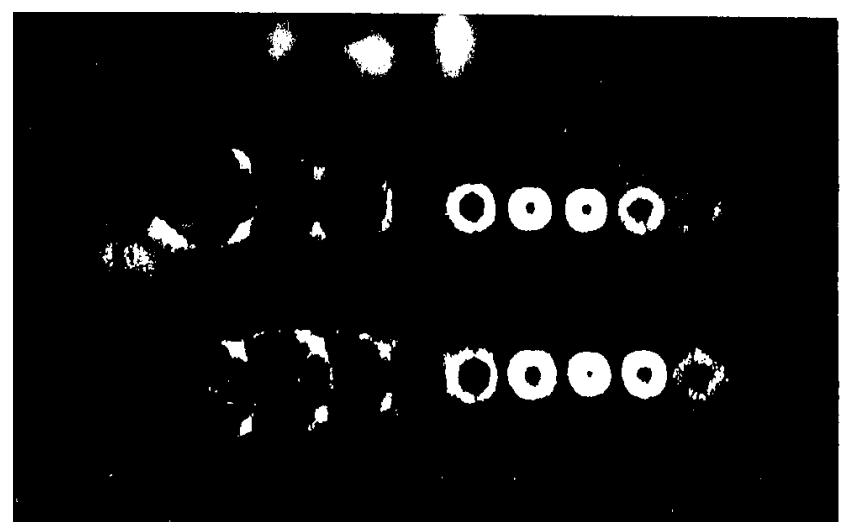

Figure 5. Plasma implosion cross section obtained by IMA$\mathrm{CON}$ framing camera with $0.2 \mu \mathrm{s}$ interval with $3.3 \mathrm{~m}$ Torr filling pressure (Hydrogen). 


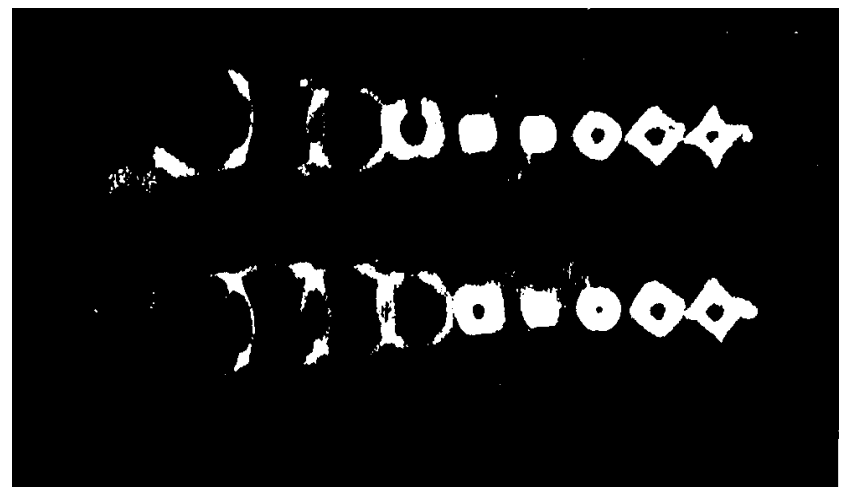

Figure 6. Azimuthal rotation of the plasma mode taken from IMACON framing camera with $0.2 \mu \mathrm{s}$ interval ( $\mathrm{Hy}-$ drogen, $7.5 \mathrm{~m}$ Torr).

In Fig. 6, it starts the appearance of the $m=4$ mode. The filling pressure in this case is $7.5 \times 10^{-3}$ Torr and the preionization time is $16 \mu \mathrm{s}$ (two periods).

In Fig. 7, we show the case with same preionization sequence as in Figure 6, but with a Hydrogen filling pressure of $10 \times 10^{-3}$ Torr, where the start of framing pictures were delayed by $2 \mu \mathrm{s}$.

One can notice from the last two framing pictures that during the implosion phase the plasma is circular and it becames more square shaped and rotates around the axis at near maximum compression. After this phase, the plasma rotation is stopped by the leak of particles from the square corners touching the wall, but still mantaining the mode structure. The total time of the sequence of events leading to an square plasma is about $2 \mu \mathrm{s}$.

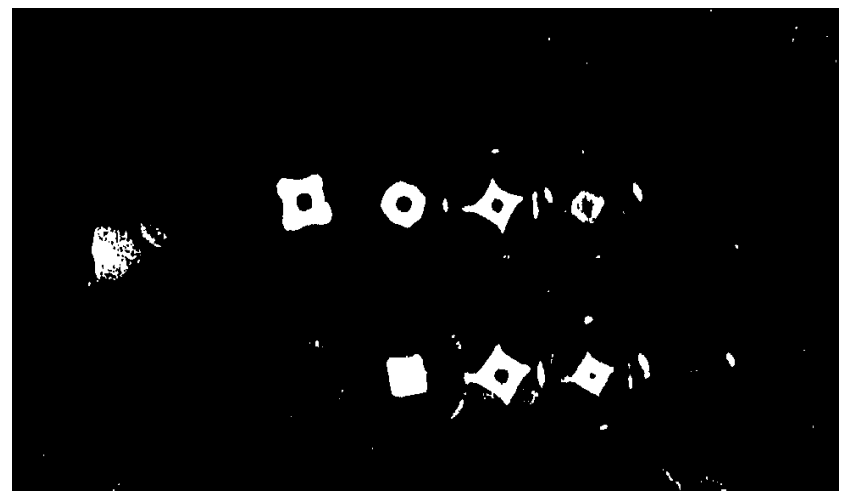

Figure 7. Azimuthal rotation of the plasma mode taken from IMACON framing camera with $0.5 \mu$ s interval (Hydrogen, 10 mTorr).

The plasma azimuthal rotation inferred from Fig. 7 before the wall touching is $1.4 \times 10^{6} \mathrm{rad} / \mathrm{s}$, as obtained from the square plasma rotation during two sequences of frame pictures.

\section{The rotational modes}

We use our non resistive MHD model to calculate the rotational modes using TC-1 parameters. We solved equation (13) using typical field reversed equilibrium magnetic profile, to analyze the $m=2$ and $m=4$ modes. Taking the magnetic field at the edge $B\left(r_{w}\right)$ to be $3.5 \mathrm{kG}$, the separatrix radius $r_{0}$ be $3.5 \mathrm{~cm}$, the peak plasma density $n\left(r_{0}\right)$ to be $10^{15} \mathrm{~cm}^{-3}$, the Alfvén transit time $\tau_{A}$ is $0.1 \mu \mathrm{s}$. Since the measured plasma rotation is $\Omega^{\prime}=1.4 \times 10^{6} \mathrm{rad} / \mathrm{s}$, the normalized rotation $\Omega_{0}$ is of the order of 0.2 . Estimating the plasma length $L$ to be $20 \mathrm{~cm}$, and considering $\lambda=2 L$, the normalized wavevector $k r_{0}$ is 0.5 . The real and imaginary parts of the eigenfrequency $\omega=\omega^{\prime} / \Omega^{\prime}=\omega^{\prime} \tau_{A} / \Omega_{0}$ are plotted in Fig. 8, for the $m=2$ mode, as a function of the wavevector $k r_{0}$ showing a decreasing $\operatorname{Im}\left(\omega^{\prime}\right) \tau_{A} / \Omega_{0}$ and an increasing $\operatorname{Re}\left(\omega^{\prime}\right) \tau_{A} / \Omega_{0}$, and we define $\gamma=\operatorname{Im}(\omega)$. At $k r_{0} \rightarrow 0$, Fig. 8 shows the $\operatorname{Re}(\omega) / \gamma=1$ limit, for the first radial mode, which agrees with the earlier work of Freidberg and Wesson [1]. Firstly, we note that $\operatorname{Re}(\omega)$ is different from $m \Omega$ in the long wavelength and small $k r_{0}$ limit. This shows a fundamental error in boundary layer analysis which assumes the marginal stability expansion $\omega=m \Omega+i \gamma$ in solving the $k r_{0}$ limit. The expansion would be adequate in the short wavelength limit. Second, the result of [1] is based on a $\theta$-pinch with parallel bias where $\mathrm{B}(\mathrm{r})$ is nonzero everywhere. They used an incompressible perturbed fluid model and assumed a more restricted expansion of the perturbed variables $(\nabla \cdot \vec{\xi}=0)$. In our case we assume compressibility and the general expansion of the perturbed quantities obtaining the same general eigenmode equation as in [4].

The eigenfrequency $\omega^{\prime} \tau_{A} / \Omega_{0}$ is plotted as a function of rotation $\Omega_{0}$ in Fig. $9\left(\Omega_{0}=0.20\right)$, for the $m=2$ mode, showing that $\gamma$ goes to zero as rotation goes to zero. The displacement $\xi_{r}$, real and imaginary parts, are plotted in Figs. 10 and 11 respectively as a function of radius $r / r_{0}$. For the $m=4$ mode, Fig. 12 shows the real and imaginary parts of the frequency $\omega^{\prime} \tau_{A} / \Omega_{0}$ as a function of the wavevector $k r_{0}$. The corresponding displacement $\xi_{r}$, real and imaginary parts, is shown in Figs. 13 and 14 as a function of radius $r / r_{0}$. In Figure 15 , the eigenfrequency $\omega^{\prime} \tau_{A} / \Omega_{0}$ is plotted against the 
rotation $\Omega_{0}$ which shows again that $\gamma \rightarrow 0$ in the zero rotation limit.

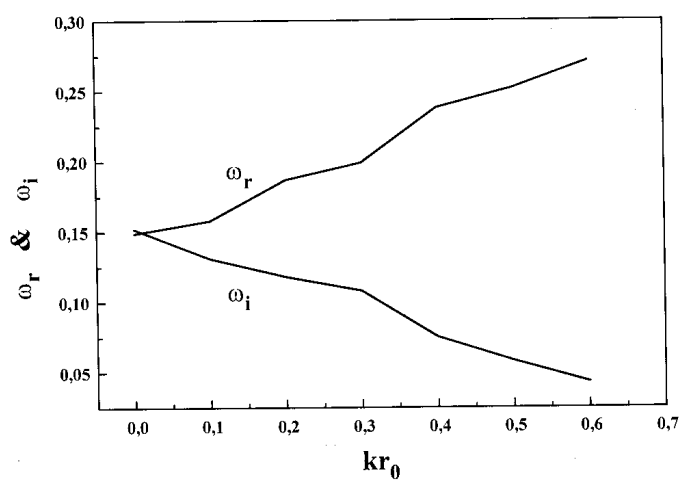

Figure 8. Real and imaginary parts of the eigenfrequency $\omega$ for $m=2$ radial modes as a function of wavevector $k r_{0}\left(\Omega_{0}=0.20\right)$.

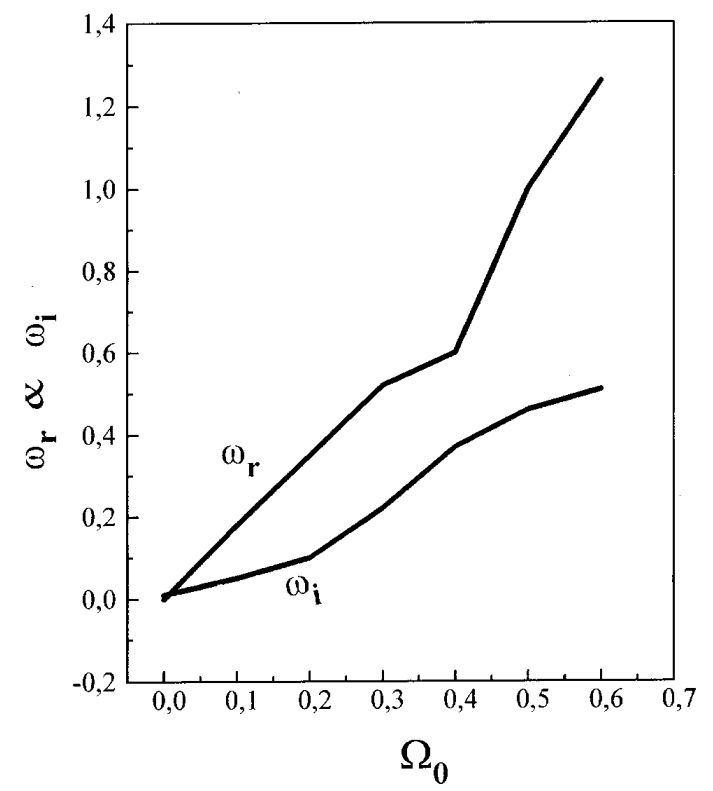

Figure 9. Real and imaginary parts of the eigenfrequency $\omega$ for $m=2$ radial modes as a function of the rotation $\Omega\left(k r_{0}=0.5\right)$.

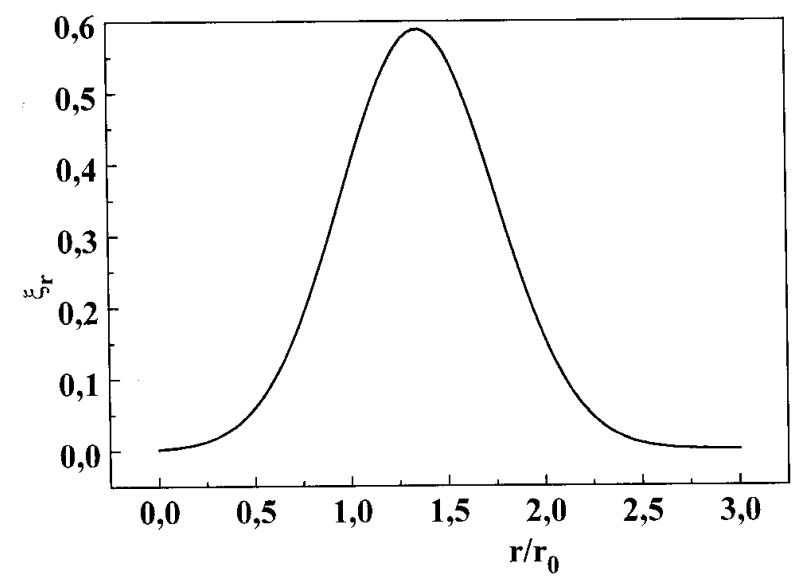

Figure 10. Real part of the eigenfunction $\xi_{r}$ for $\mathrm{m}=2$ as a function of radius $r / r_{0}\left(\Omega_{0}=0.20\right.$ and $\left.\left.k r_{0}=0.5\right)\right)$.

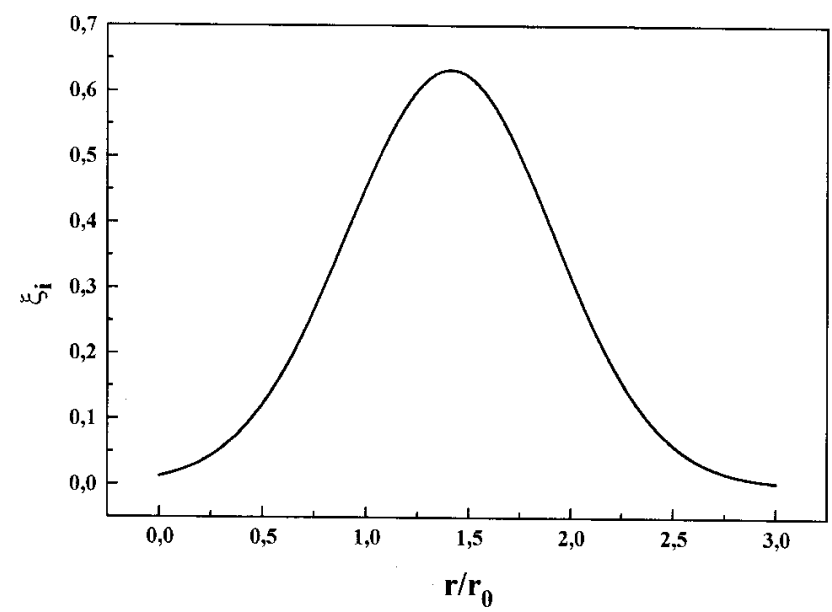

Figure 11. Imaginary part of the eigenfunction $\xi_{r}$ for $\mathrm{m}=2$ as a function of radius $r / r_{0}\left(\Omega_{0}=0.20\right.$ and $\left.k r_{0}=0.5\right)$.

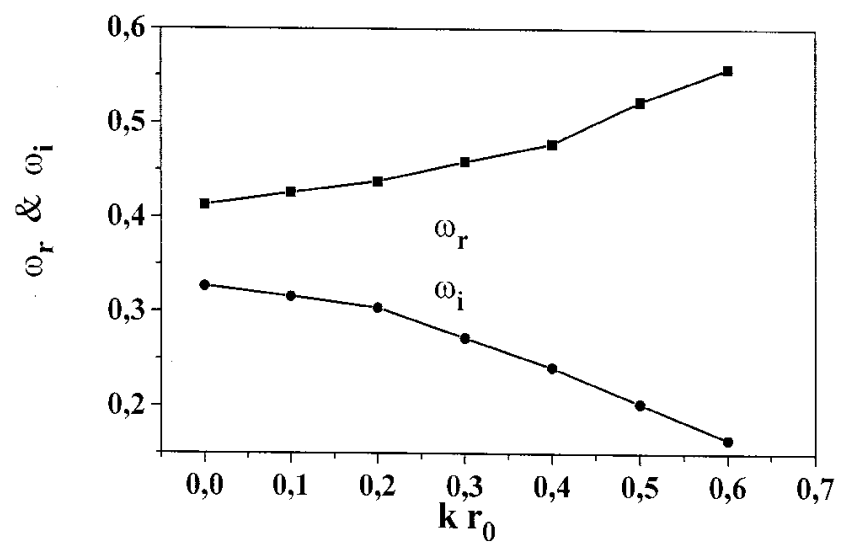

Figure 12. Real and imaginary parts of the eigenfrequency $\omega$ for $m=4$ radial modes as a function of the wavevector $k r_{0}\left(\Omega_{0}=0.20\right)$.

Taking $\Omega_{0}=0.20$ for the TC-1 machine, Fig.15 gives $\operatorname{Im}\left(\omega^{\prime}\right) \tau_{A} / \Omega_{0}=0.30$ for the $\mathrm{n}=0$ radial mode (fundamental mode). This corresponds to $\gamma^{-1}$ of the order of $1.3 \mu \mathrm{s}$ and the measured growth time is of the order of $2.0 \mu \mathrm{s}$. The appearance of the $m=4$ mode rather than the $m=2$ mode in the $\mathrm{TC}-1$ can be justified by the larger growth rates in Fig. 15 than those in Fig.9. 


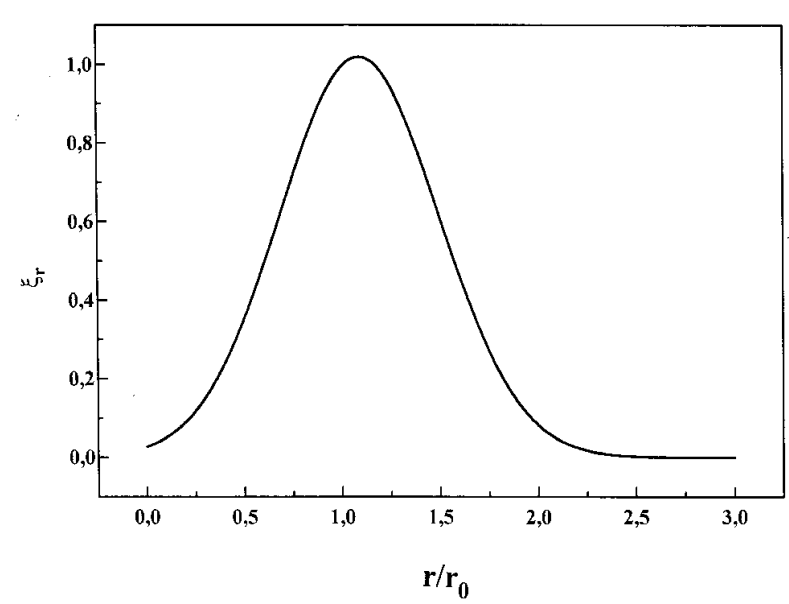

Figure 13. Real part of the eigenfunction $\xi_{r}$ for $m=4$ (fundamental mode $n=0)$ as a function of $\mathrm{r}\left(\Omega_{0}=0.20\right.$ and $\left.k r_{0}=0.5\right)$.

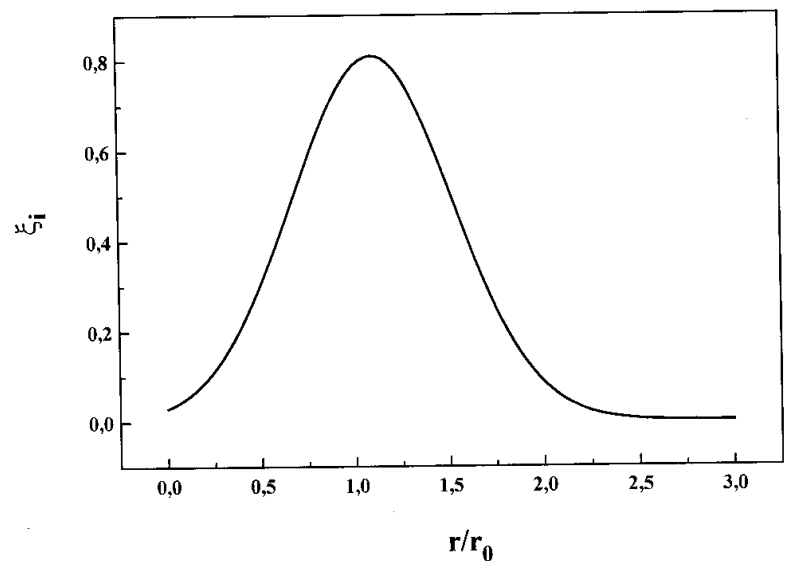

Figure 14. Imaginary part of the eigenfunction $\xi_{r}$ for $\mathrm{m}=4$ as a function of radius $r / r_{0}\left(\Omega_{0}=0.20\right.$ and $\left.k r_{0}=0.5\right)$.

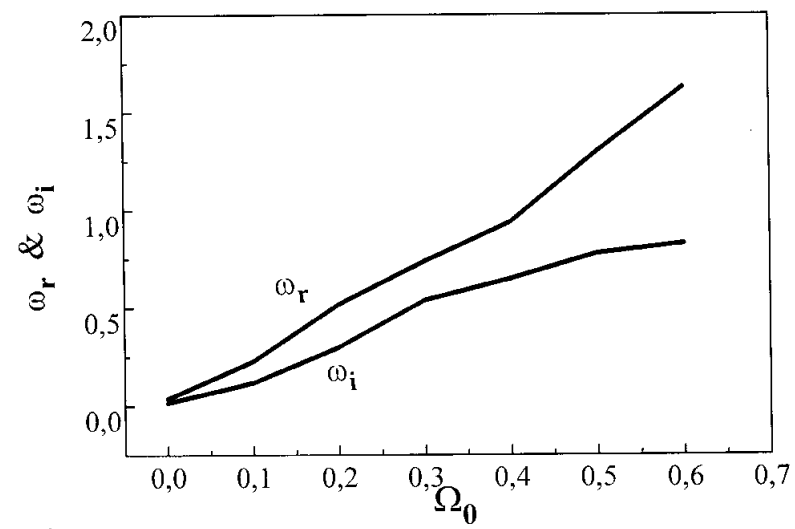

Figure 15. Real and imaginary parts of the eigenfrequency $\omega$ for $m=4$ radial modes as a function of rotation $\Omega$ and with $k r_{0}=0.5$.

\section{Conclusions}

We used a one dimensional MHD model to study the rotational instability in FRC plasmas taking into account the plasma compressibility and comparing our results with the TC-1 machine of Unicamp.

Although the $m=2$ mode is commonly observed in many machines, [12], we have only seen the $m=4$ mode in TC-1. However, the presence of this mode is compatible with the hybrid code of Harned [6], which has predicted high $\mathrm{m}$ modes. We emphasize that the appearance of the $\mathrm{m}=4$ mode in $\mathrm{TC}-1$ depends decisively on the timing of the capacitor banks, whereas filling pressure plays a less critical role.

\section{Acknowledgements}

This work was supported by the Conselho Nacional de Desenvolvimento Científico e Tecnológico (CNPq), the Financiadora de Estudos e Projetos (FINEP), and the Fundação de Amparo a Pesquisa do Estado de São Paulo (FAPESP).

\section{References}

[1] J. P. Freidberg and J. A. Wesson, Phys. of Fluids 13, 1117 (1970).

[2] J. L. Schwarzmeier, D. C. Barnes, D.W. Hewett, C. E. Seyler, A. I. Shestakov and R. L. Spencer, Phys. of Fluids 26, 1295 (1983).

[3] D. C. Barnes and D. V. Anderson, Phys. Rev. Letters 46, 1337 (1981).

[4] E. Frieman and M. Rotenberg, Rev. of Mod. Phys. 32, $898(1960)$

[5] T. Ishimura, Phys. of Fluids 27, 2139 (1984).

[6] D. Harned, Phys. of Fluids 26, 1320 (1983)

[7] E. Hameiri, Phys. of Fluids 26, 230 (1983).

[8] Y. Ito, M. Tanjyo, S. Ohi, S. Goto and T. Ishimura, Phys. of Fluids 30, 168 (1987).

[9] B. Coppi, R. M. O. Galvão, R. Pellat, M. Rosenbluth and P. Rutherford, Sov. J. of Plasma Phys. 2, 533 (1976).

[10] R. M. O. Galvão and M. A. M. Santiago, Phys. of Fluids 24, 661 (1981).

[11] D. O. Campos, M. Machida, S. V. Lebedev, M. Kantor, S. A. Moshkalyov and L. A. Berni, Braz. J. Phys. 26, 747 (1996).

[12] A. L. Hoffman, J. T. Slough and D. G. Harding, Phys. of Fluids 26, 1226 (1983). 